\title{
Correction to: Overexpression of CAV3 facilitates bone formation via the Wnt signaling pathway in osteoporotic rats
}

\section{Run-Bao Yang ${ }^{1} \cdot$ Feng-Fei Lin $^{2} \cdot$ Jun Yang ${ }^{1} \cdot$ Bin Chen $^{2} \cdot$ Ming-Hua Zhang ${ }^{1} \cdot$ Qiao-Ping Lu ${ }^{1} \cdot$ Bo Xiao ${ }^{1}$ Yan Liu ${ }^{1}$. Ke Zheng ${ }^{2} \cdot$ Yong-Rong Qiu ${ }^{3}$}

Published online: 18 September 2020

(c) Springer Science+Business Media, LLC, part of Springer Nature 2020, corrected publication 2020

Correction to: Endocrine (2019) 63:639-650

https://doi.org/10.1007/s12020-018-1803-1
The original version of this article unfortunately contained a mistake in Fig. 10. The correct version of Fig. 10 is given below.

These authors are regarded as co-first authors: Run-Bao Yang, Feng-Fei Lin.

The original article can be found online at https://doi.org/10.1007/ s12020-018-1803-1.

Yong-Rong Qiu

qyr2299811@163.com

1 Department of Orthopedics and Traumatology, Longyan First Hospital, Longyan 364000, P.R. China

2 Department of Orthopedic Surgery, Fuzhou Second Hospital, Fuzhou 350007, P.R. China

3 Department of Orthopaedics Surgery, Longyan First Hospital, Longyan 364000, P.R. China 

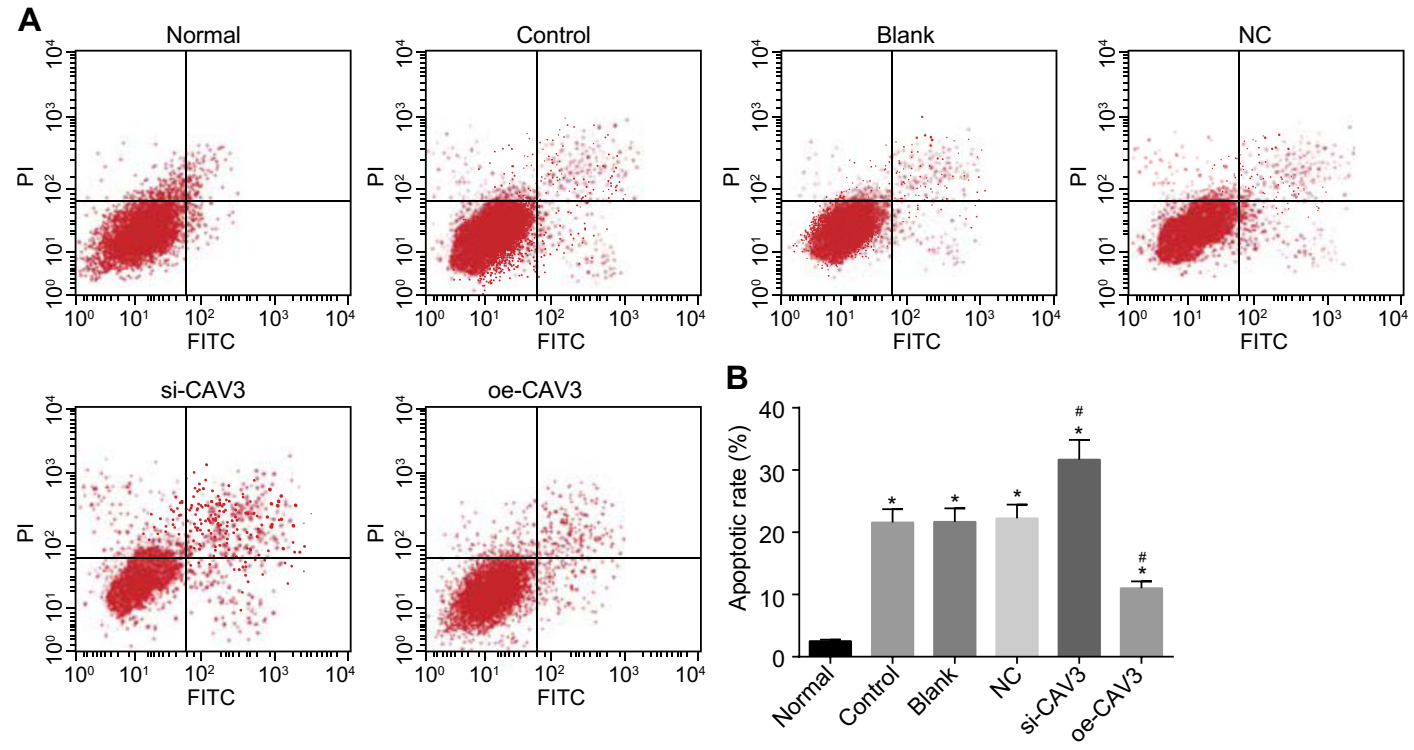

Fig. 10 Annexin V-FITC/PI double standard staining demonstrated that cell apoptosis was promoted following the transfection of siCAV3. a cell apoptosis profile in six groups; b histogram of apoptosis in six groups; $* p<0.05$ vs. the normal group; ${ }^{*} p<0.05$ vs. the control group. Measurement data were expressed as the mean \pm standard deviation, one-way analysis of variance was used for comparisons among multiple groups. The experiment was conducted independently 3 times 